\title{
Interaction induced quasi-particle spectrum in holography
}

\author{
Geunho Song, ${ }^{a}$ Yunseok Seo, ${ }^{b}$ Keun-Young Kim ${ }^{c}$ and Sang-Jin Sin ${ }^{a}$ \\ ${ }^{a}$ Department of Physics, Hanyang University, \\ Seoul 04763, Korea \\ ${ }^{b}$ GIST college, Gwangju Institute of Science and Technology, \\ Gwangju 61005, Korea \\ ${ }^{c}$ School of Physics and Chemistry, Gwangju Institute of Science and Technology, \\ Gwangju 61005 Korea \\ E-mail: sgh8774@gmail.com, yseo@gist.ac.kr, fortoe@gist.ac.kr, \\ sjsin@hanyang.ac.kr
}

ABSTRACT: It is often said that interactions destroy the particle nature of excitations. We report that, in holographic theory adding interaction term can create a new quasi particle spectrum, on the contrary. We show this by calculating the optical conductivity in a model with exact background solution and finding a new quasi-particle spectrum. Such new poles are consequence of some non-minimal interaction like Chern-Simon term. We also point out that the origin of the new peak in our example is the vortex formation by the anomalous magnetic moment induced by the interaction term.

KEYwORDS: Holography and condensed matter physics (AdS/CMT), AdS-CFT Correspondence, Gauge-gravity correspondence

ARXIV EPRINT: 1907.06188 


\section{Contents}

1 Introduction 1

2 Setup and methods 3

2.1 Action and background geometry 3

2.2 Linearized equations of motion and the on-shell action 4

$\begin{array}{lll}2.3 & \text { Optical conductivities } & 7\end{array}$

3 Interaction induced quasi-particles in AC conductivity 10

3.1 Appearance of a new quasi particle at $\mu=H=0 \quad 10$

3.2 Pinning peak vs. Cyclotron peak: $\mu=0, H \neq 0 \quad 11$

3.3 Drude vs. Pinning peak: $\mu \neq 0$ and $H=0 \quad 13$

$\begin{array}{ll}3.4 \text { Origin of pinning pole } & 15\end{array}$

4 Discussion $\quad 16$

\section{Introduction}

The effects of an interaction in weakly interacting theory are of two folds: one is to smooth out the particle character of the basic excitations and the other is to mix and deform spectral curves which exist before we add the interaction term. On the other hand, for the Lagrangian with 'free' holographic bulk field, particle spectrum of boundary dual theory is already washed out at least partially through the minimal coupling of the field to the bulk gravity. This can be demonstrated in the bulk fermion's Green function, $G_{R} \sim$ $k^{2 m-1} \gamma \cdot k[1]$. Then what is the role of the bulk interaction term?

In this paper, we will show that the bulk interaction term can create a new pole in the Green function for the system where original spectrum is almost featureless. This is somewhat opposite phenomena to that of weakly interacting system. In the fermion spectral function with bulk mass $m$, there was a hint already: in search of the gap creation using the Pauli term, $p \bar{\psi} \gamma^{\mu \nu} F_{\mu \nu} \psi$ [2], it was observed [3] that a new branch of spectral curve is created after adding the Pauli term. It was based on the parameter regime of $m \approx-0.5$ where the spectral function mimics that of the weakly interacting theory. However, even for the regime $m \approx 0.5$ where particle natures are washed out, a new branch with a sharp particle feature appears for a large enough $p$, which is unlikely to happen in weakly interacting systems. See the figure 1(a) and (b) with and without the Pauli term.

Here, we want to discuss the phenomena in the context of transport so that we can discuss phenomena like insulator to metal transition more directly. For this reason, we consider the optical conductivity by considering the bulk field $A_{\mu}$ which is dual to the current $J_{\mu}$. The optical conductivity can also be considered as a measure of spectral 


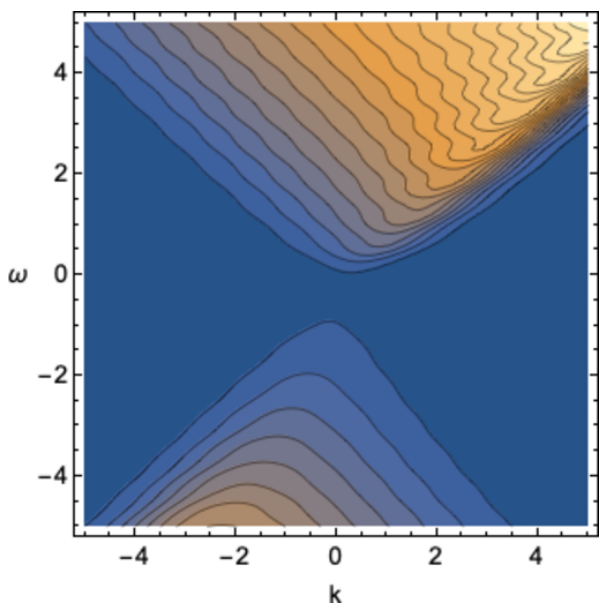

(a) Without the Pauli term

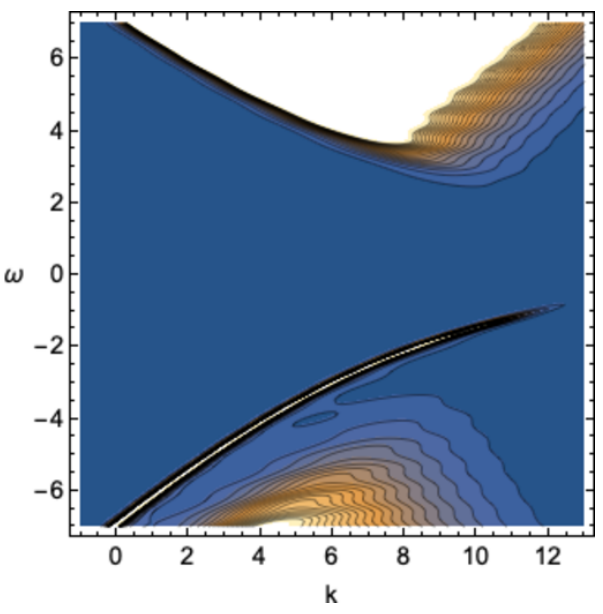

(b) With the Pauli term

Figure 1. Fermion spectral functions. (a) Before the Pauli term is added. No particle nature. (b) After the Pauli term is added, a new branch appears, implying new quasi-particle creation. The background used is the RN black hole with chemical potential $\mu=\sqrt{3}$, temperature $T=0.1$ and $p=0$ and 10 for (a) and (b) respectively.

function of vector particles in the boundary. We use a background that has analytical expression. This is a model $[4,5]$ where a charged black hole is deformed by introducing an interaction term describing the magnetic impurities coupled to the 'instanton density' $F \wedge F$. A quasi particle peak develops as we increase the strength of interaction of the magnetic impurity and the peak becomes sharper for a larger coupling. This phenomena can be described as "bulk interaction term is the dual of the boundary quasi-particle".

The study of the character change of holographic system induced by the interaction term has some history, although some of them goes opposite way from our example by killing the existing pole. In [6-12], it was noted that adding the Chern-Simon term $A \wedge F \wedge F$ can lead to the charge density wave instability. In [13-18], it was reported that a gap in optical conductivity can be created in the presence of a Chern-Simon like term $A_{2} \wedge F_{2} \wedge F_{1}$ if $A_{2}$ is magnetically sourced and the physics was identified as metal-insulator transition, which is opposite to what we report here. Therefore the role of our interaction term is opposite to the usual Chern Simon case where it creates charge density wave (CDW) gap, that is, it kills the Drude pole, while here it is acting as a creator of new pole. In the discussion section, we discuss the reason why in our example there is no instability.

More recently, in [19-22], a shifted peak in optical conductivity was interpreted as the pinning effect [23-25]. Namely, if we consider the acoustic phonon as a Goldstone boson of translational symmetry breaking and asks what is the fate of it when we add small explicit symmetry breaking, they suggest that it disappears by getting mass. Both insulator to metal transition and the pinning effect share the creation of new peak by interaction and can be viewed in the framework of spectral reconfiguration by the interaction although their phenomena belongs to completely different classes. 
The rest of the paper goes as follows. in section 2 after describing the methods, in section 3 we will calculate the AC conductivity as a spectral measure to demonstrate the appearance of new quasi-particle pole and we discuss the origin of the new quasi particle peak. We conclude with discussions at section 4 .

\section{Setup and methods}

In this section we describe the basic set up and calculational methods for optical conductivity. We adopted the basic scheme of [26] for our purpose. The readers who are not interested in technical details can go to the section 3 directly.

\subsection{Action and background geometry}

We start from the Einstein-Maxwell action with two massless scalars for momentum relaxation [27], one of which is coupled with the gauge instanton density on 4-d manifold $\mathcal{M}$ with boundary $\partial \mathcal{M}$.

$$
\begin{aligned}
2 \kappa^{2} S_{0}= & \int_{\mathcal{M}} d^{4} x \sqrt{-g}\left\{R+\frac{6}{L^{2}}-\frac{1}{4} F^{2}-\sum_{I, a=1,2} \frac{1}{2}\left(\partial \chi_{I}^{(a)}\right)^{2}\right\} \\
& -\frac{q_{\chi}}{16} \int_{\mathcal{M}}\left(\partial \chi_{I}^{(2)}\right)^{2} \epsilon^{\mu \nu \rho \sigma} F_{\mu \nu} F_{\rho \sigma}-2 \int_{\partial \mathcal{M}} d^{3} x \sqrt{-\gamma} K \\
S_{c}= & -\int_{\partial \mathcal{M}} d^{3} x \sqrt{-\gamma}\left(\frac{4}{L}+R[\gamma]-\sum_{I, a=1,2} \frac{L}{2} \nabla \chi_{I}^{(a)} \cdot \nabla \chi_{I}^{(a)}\right),
\end{aligned}
$$

where $q_{\chi}$ is a coupling, and $\kappa^{2}=8 \pi G$ and $L$ is the AdS radius and we set $2 \kappa^{2}=L=1$. $S_{c}$ is the counter term for holographic renormalization. The finite renormalized on-shell action is

$$
S_{\text {ren }}=\lim _{\Lambda \rightarrow \infty}\left(S_{0}+S_{c}\right)_{\text {on-shell }} \text {. }
$$

The corresponding equations of motion are given by

$$
\begin{gathered}
\partial_{\mu}\left(\sqrt{-g} g^{\mu \nu} \sum_{I}\left(\partial_{\nu} \chi_{I}^{(a)}\right)\right)+\frac{q_{\chi}}{8} \partial_{\mu}\left(\epsilon^{\rho \sigma \lambda \gamma} F_{\rho \sigma} F_{\lambda \gamma} g^{\mu \nu} \sum_{I, a}\left(\partial_{\nu} \chi_{I}^{(a)} \delta_{2 a}\right)\right)=0 \\
\partial_{\mu}\left(\sqrt{-g} F^{\mu \nu}+\frac{q_{\chi}}{4} g^{\rho \sigma} \sum_{I, a}\left(\partial_{\rho} \chi_{I}^{(a)} \partial_{\sigma} \chi_{I}^{(a)} \delta_{2 a}\right) \epsilon^{\alpha \beta \mu \nu} F_{\alpha \beta}\right)=0 \\
R_{\mu \nu}-\frac{1}{2} g_{\mu \nu}\left[R+6-\frac{1}{4} F^{2}-\sum_{I, a} \frac{1}{2}\left(\partial \chi_{I}^{(a)}\right)^{2}\right]-\frac{1}{2} F_{\mu}^{\rho} F_{\rho \nu}-\frac{1}{2} \sum_{I, a}\left(\partial_{\mu} \chi_{I}^{(a)}\right)\left(\partial_{\nu} \chi_{I}^{(a)}\right) \\
-\frac{1}{\sqrt{-g}} \frac{q_{\chi}}{16} \sum_{I, a}\left(\partial_{\mu} \chi_{I}^{(a)}\right)\left(\partial_{\nu} \chi_{I}^{(a)}\right) \delta_{2 a} \epsilon^{\rho \sigma \lambda \gamma} F_{\rho \sigma} F_{\lambda \gamma}=0 .
\end{gathered}
$$


With the following ansatz,

$$
\begin{aligned}
A & =a(r) d t+\frac{1}{2} H(x d y-y d x), \\
\chi_{I}^{(1)} & =\left(\begin{array}{c}
\alpha x \\
\alpha y
\end{array}\right), \quad \chi_{I}^{(2)}=\left(\begin{array}{c}
\beta x \\
\beta y
\end{array}\right) \\
d s^{2} & =-U(r) d t^{2}+\frac{d r^{2}}{U(r)}+r^{2}\left(d x^{2}+d y^{2}\right),
\end{aligned}
$$

we found the exact solutions as follows:

$$
\begin{aligned}
U(r) & =r^{2}-\frac{\alpha^{2}+\beta^{2}}{2}-\frac{m_{0}}{r}+\frac{q^{2}+H^{2}}{4 r^{2}}+\frac{\beta^{4} H^{2} q_{\chi}^{2}}{20 r^{6}}-\frac{\beta^{2} H q q_{\chi}}{6 r^{4}}, \\
a(r) & =\mu-\frac{q}{r}+\frac{\beta^{2} H q_{\chi}}{3 r^{3}}
\end{aligned}
$$

where $\mu$ is the chemical potential, $q$ and $m_{0}$ are determined by the conditions $A_{t}\left(r_{0}\right)=$ $U\left(r_{0}\right)=0$ at the black hole horizon $\left(r=r_{0}\right) . q$ is the conserved $\mathrm{U}(1)$ charge density and $\alpha, \beta$ correspond to the non-magnetic impurity and magnetic impurity density respectively.

$$
\begin{aligned}
q & =\mu r_{0}+\frac{1}{3} \theta H \quad \text { with } \quad \theta=\frac{\beta^{2} q_{\chi}}{r_{0}^{2}} \\
m_{0} & =r_{0}^{3}\left(1+\frac{r_{0}^{2} \mu^{2}+H^{2}}{4 r_{0}^{4}}-\frac{\alpha^{2}+\beta^{2}}{2 r_{0}^{2}}\right)+\frac{\theta^{2} H^{2}}{45 r_{0}}
\end{aligned}
$$

The temperature of the boundary system can be derived from the Hawking temperature in the bulk,

$$
4 \pi T=U^{\prime}\left(r_{0}\right)=3 r_{0}-\frac{1}{4 r_{0}^{3}}\left[H^{2}+2 r_{0}^{2}\left(\alpha^{2}+\beta^{2}\right)+(q-H \theta)^{2}\right]
$$

and the entropy is given by the area of black hole horizon as usual

$$
s=4 \pi r_{0}^{2}
$$

\subsection{Linearized equations of motion and the on-shell action}

To compute the optical conductivity, we need to consider small perturbations around the background (2.7) and (2.8) to use linear response theory. We turn on small fluctuations $\delta g_{t i}, \delta A_{i}$, and $\delta \chi_{i}$ with $i=x, y$, around the background at zero momentum $(\vec{k}=0)$. The fields $\delta g_{t i}$ and $\delta A_{i}$ are related to the temperature gradient and the electric field respectively. Now we add $\delta \chi_{i}$ for momentum relaxation for finite conductivity. For optical conductivities, we should consider a gauge $\delta g_{r i}=0$, and this gauge does not change any physical results. Indeed, the zero frequency limit of optical conductivity in this gauge $\left(\delta g_{r i}=0\right)$ is consistent with the DC conductivity which is analytically obtained with non-trivial gauge of $\delta g_{r i}$. 
These fields, $\delta g_{t i}, \delta A_{i}$ and $\delta \chi_{i}^{(a)}$, can be expressed in momentum space as

$$
\begin{aligned}
\delta A_{i} & =\int_{-\infty}^{\infty} \frac{d \omega}{2 \pi} e^{-i \omega t} a_{i}(r), \\
\delta g_{t i} & =\int_{-\infty}^{\infty} \frac{d \omega}{2 \pi} e^{-i \omega t} r^{2} h_{t i}(r) \\
\delta \chi_{i}^{(1)} & =\int_{-\infty}^{\infty} \frac{d \omega}{2 \pi} e^{-i \omega t} \phi_{i}(r) \\
\delta \chi_{i}^{(2)} & =\int_{-\infty}^{\infty} \frac{d \omega}{2 \pi} e^{-i \omega t} \psi_{i}(r),
\end{aligned}
$$

where $h_{t i}$ goes to constant at the boundary $(r \rightarrow \infty)$ because of $r^{2}$ in the metric fluctuations (2.15) and we set $r_{0}=1$ for convenience of numerical analysis. From the equations of motion (2.4)-(2.6), the linearised equations in momentum space are given by the following 8 equations.

- Einstein equations:

$$
\begin{aligned}
0= & h_{t i}^{\prime \prime}+\frac{4}{r} h_{t i}^{\prime}+\frac{\left(q-\theta H / r^{2}\right)}{r^{4}} a_{x}^{\prime}-\frac{H^{2}}{r^{4} U} h_{t i}-\frac{i \alpha \omega}{r^{2} U} \phi_{i} \\
& -\frac{\left(\beta^{2} r^{4}-q \theta H-\theta^{2} H^{2} / r^{2}\right)\left(\beta h_{t i}+i \omega \psi_{i}\right)}{\beta r^{6} U}-\frac{\alpha^{2}}{r^{2} U} h_{t i}-\epsilon_{i j} \frac{i \omega H}{r^{4} U} a_{j} \\
0= & h_{t i}^{\prime}+\epsilon_{i j} \frac{i U H}{r^{4} \omega} a_{j}^{\prime}+\frac{i \beta U\left(r^{6}-q_{\chi} q H r^{2}+q_{\chi}^{2} H^{2} \beta^{2}\right)}{r^{8} \omega} \psi_{i}^{\prime}+\frac{i \alpha U}{r^{2} \omega} \phi_{i}^{\prime} \\
& +\frac{\left(q r^{2}-q_{\chi} H \beta^{2}\right)\left(\omega a_{x}+i H \epsilon_{i j} h_{t j}\right)}{r^{6} \omega}
\end{aligned}
$$

- Maxwell equations:

$$
0=a_{i}^{\prime \prime}+\frac{U^{\prime}}{U} a_{i}^{\prime}+\frac{\left(q-\theta H / r^{2}\right)}{U} h_{t i}^{\prime}+\frac{\omega^{2}}{U^{2}} a_{i}+\epsilon_{i j}\left(\frac{2 i \theta \omega}{r^{3} U} a_{j}+\frac{i H \omega}{U^{2}} h_{t j}\right)+\frac{2 \theta H}{r^{3} U} h_{t i}
$$

- Scalar equations:

$$
\begin{aligned}
0= & \phi_{i}^{\prime \prime}+\left(\frac{2}{r}+\frac{U^{\prime}}{U}\right) \phi_{i}^{\prime}-\frac{i \alpha \omega}{U^{2}} h_{t x}+\frac{\omega^{2}}{U} \phi_{i} \\
0= & \left(1-\frac{q \theta H}{\beta^{2} r^{4}}+\frac{\theta^{2} H^{2}}{\beta^{2} r^{6}}\right) \psi_{i}^{\prime \prime}+\left(\frac{2}{r}+\frac{2 q \theta H}{\beta^{2} r^{5}}-\frac{4 H^{2} \theta^{2}}{\beta^{2} r^{7}}+\frac{U^{\prime}}{U}-\frac{q \theta H U^{\prime}}{\beta^{2} r^{4} U}+\frac{H^{2} \theta^{2} U^{\prime}}{\beta^{2} r^{6} U}\right) \psi_{i}^{\prime} \\
& +\frac{\omega\left(\beta^{2} r^{4}-q H \theta-\theta^{2} H^{2} / r^{2}\right)\left(\omega \psi_{i}-i \beta h_{t i}\right)}{\beta^{2} r^{4} U^{2}}
\end{aligned}
$$

Among these 10 equations, only 6 are independent. Indeed, differentiating (2.19) with respect to $r$ and putting background solution into the equation, then we can obtain (2.18). We need to find the boundary conditions to solve these equations. There are two boundary conditions, which are the incoming boundary conditions at the horizon and the Dirichlet boundary conditions at the boundary. Near the black hole horizon $(r \rightarrow 1)$ the solutions 
are expanded as

$$
\begin{aligned}
h_{t i} & =(r-1)^{\nu_{ \pm}+1}\left(h_{t i}^{(I)}+h_{t i}^{(I I)}(r-1)+\cdots\right), \\
a_{i} & =(r-1)^{\nu_{ \pm}}\left(a_{i}^{(I)}+a_{i}^{(I I)}(r-1)+\cdots\right), \\
\phi_{i} & =(r-1)^{\nu_{ \pm}}\left(\phi_{i}^{(I)}+\phi_{i}^{(I I)}(r-1)+\cdots\right) \\
\psi_{i} & =(r-1)^{\nu_{ \pm}}\left(\psi_{i}^{(I)}+\psi_{i}^{(I I)}(r-1)+\cdots\right)
\end{aligned}
$$

where

$$
\nu_{ \pm}= \pm 36 i \omega /\left(H^{2}\left(9+4 q_{\chi}^{2} \beta^{4}\right)-12 q_{\chi} H \beta^{2} \mu+9\left(-12+2 \beta^{2}+\mu^{2}\right)\right)
$$

and the incoming boundary condition corresponds to taking $\nu_{+}$and discarding $\nu_{-}$. It turns out that only 6 coefficients, $a_{i}^{(I)}, \psi_{i}^{(I)}$ and $\phi_{i}^{(I)}$, of horizon behavior of the solutions can be chosen independently because $h_{t i}^{(I)}$ and all other higher order coefficients can be determined by them.

The asymptotic solutions near the boundary $(r \rightarrow \infty)$ can be written by

$$
\begin{aligned}
h_{t i} & =h_{t i}^{(0)}+\frac{1}{r^{2}} h_{t i}^{(2)}+\frac{1}{r^{3}} h_{t i}^{(3)}+\cdots, \\
a_{i} & =a_{i}^{(0)}+\frac{1}{r} a_{i}^{(1)}+\cdots, \\
\phi_{i} & =\phi_{i}^{(0)}+\frac{1}{r^{2}} \phi_{i}^{(2)}+\frac{1}{r^{3}} \phi_{i}^{(3)}+\cdots, \\
\psi_{i} & =\psi_{i}^{(0)}+\frac{1}{r^{2}} \psi_{i}^{(2)}+\frac{1}{r^{3}} \psi_{i}^{(3)}+\cdots .
\end{aligned}
$$

With the incoming boundary condition and chosen free parameters $a_{i}^{(I)}, \phi_{i}^{(I)}$, and $\psi_{i}^{(I)}$, we can numerically determine the leading and subleading terms by solving linearised equations of motion (2.18)-(2.22). The leading terms play the role of sources for the operators whose expectation values are encoded in $h_{t i}^{(3)}, a_{i}^{(1)}, \phi_{i}^{(3)}$, and $\psi_{i}^{(3)}$ respectively.

We expand the renormalized action (2.3) around the modified RN- $A d S_{4}$ background (2.7)(2.8) and using the equations of motion to obtain a quadratic on-shell action:

$$
\begin{aligned}
S_{\mathrm{ren}}^{(2)}= & \lim _{\Lambda \rightarrow \infty} \frac{1}{2} \int_{r=\Lambda} d^{3} x\left[\delta \tilde{h}_{t i}\left(\left(-q+\frac{\theta H}{r^{2}}\right) \delta A_{i}+\frac{\left(\alpha \delta \dot{\chi}_{i}^{(1)}+\beta \delta \dot{\chi}_{i}^{(2)}\right) r^{2}}{\sqrt{U(r)}}\right)\right. \\
& +\left(4 r^{3}-\frac{4 r^{4}}{\sqrt{U(r)}}\right) \delta \tilde{h}_{t i}^{2}-r^{2} \delta \chi_{i}^{(1)} \delta \chi_{i}^{(1) \prime}-\frac{\left(\beta^{2} r^{4}-q \theta H+\theta^{2} H^{2} / r^{2}\right) U(r)}{\beta^{2} r^{2}} \delta \chi_{i}^{(2)} \delta \chi_{i}^{(2) \prime} \\
& +\epsilon_{i j} \frac{\left(q \theta H-\theta^{2} H / r^{2}\right) U(r)}{\beta^{2} r^{2}} \delta \chi_{i}^{(2)} \delta \chi_{j}^{(2) \prime}+\epsilon_{i j} \frac{\theta}{2 r^{2}} \delta \dot{A}_{i} \delta A_{j}-U(r) \delta A_{i} \delta A_{i}^{\prime} \\
& \left.-\frac{\alpha r^{2}}{\sqrt{U(r)}} \delta \chi_{i}^{(1)} \tilde{h}_{t i}+\frac{r^{2}}{\sqrt{U(r)}} \delta \chi_{i}^{(1)} \delta \ddot{\chi}_{i}^{(1)}-\frac{\beta r^{2}}{\sqrt{U(r)}} \delta \chi_{i}^{(2)} \tilde{h}_{t i}+\frac{r^{2}}{\sqrt{U(r)}} \delta \chi_{i}^{(2)} \delta \ddot{\chi}_{i}^{(2)}\right]
\end{aligned}
$$

where $\delta \tilde{h}_{t i}=r^{-2} \delta g_{t i}(t, r)$, dot denotes time derivative, prime denotes $r$-derivative. With the expression of fluctuation in momentum space, we can rewrite the quadratic action in 
momentum space:

$$
\begin{aligned}
S_{\text {ren }}^{(2)}= & \frac{\mathcal{V}_{2}}{2} \int_{0}^{\infty} \frac{d \omega}{2 \pi}\left(-q \bar{a}_{i}^{(0)} h_{t i}^{(0)}-2 m_{0} \bar{h}_{t i}^{(0)} h_{t i}^{(0)}+\bar{a}_{i}^{(0)} a_{i}^{(1)}-3 \bar{h}_{t i}^{(0)} h_{t i}^{(3)}+\bar{\phi}_{i}^{(0)} \phi_{i}^{(3)}+\bar{\psi}_{i}^{(0)} \psi_{i}^{(3)}\right) \\
& + \text { h.c. }
\end{aligned}
$$

where $\mathcal{V}_{2}$ is two dimensional spatial volume $\int d x d y$. It is understood that the argument of the variables is $\omega$ while that of those with the bar is $-\omega$.

\subsection{Optical conductivities}

We introduce a systematic numerical method with multi-fields and constraints for our case briefly [26]. Let us start with $n$ fields $\Phi^{a}(t, r),(a=1,2, \cdots, n)$, which are fluctuations around a background. Assume that they satisfy a set of coupled $n$ second order differential equations and all the fluctuation fields depend only on $t$ and $r$ :

$$
\Phi^{a}(t, r)=\int \frac{d \omega}{2 \pi} e^{-i \omega t} r^{p} \Phi_{\omega}^{a}(r),
$$

where $r^{p}$ is multiplied such that the solution $\Phi_{\omega}^{a}(r)$ has constant behavior at the boundary $(r \rightarrow \infty)$. For example, $p=2$ in $(2.15)$.

Near the black hole horizon $(r=1)$, solutions are expanded as

$$
\Phi^{a}(r)=(r-1)^{\nu_{a \pm}}\left(\phi^{a}+\tilde{\phi}^{a}(r-1)+\cdots\right),
$$

where we dropped the subscript $\omega$ for simplicity and \pm denotes incoming/outgoing boundary conditions respectively. For computing the retarded Green's function holographically, we should choose the incoming boundary condition. This choice eliminates the half of independent parameters from $2 n$ to $n$. And if we have $n_{c}$ constraint equations, then the number of independent parameters can be reducted by $n_{c}$. Consequently, the number of independent parameter is $n-n_{c}$ so we must choose $n-n_{c}$ initial conditions, denoted by $\phi_{\hat{i}}^{a}\left(\hat{i}=1,2, \cdots, n-n_{c}\right)$ :

$$
\left(\begin{array}{lllll}
\phi_{1}^{a} & \phi_{2}^{a} & \phi_{3}^{a} & \cdots & \phi_{n-n_{c}}^{a}
\end{array}\right)=\left(\begin{array}{cccc}
1 & 1 & 1 & \cdots \\
1 & -1 & 1 & \cdots \\
1 & 1 & -1 & \cdots \\
\vdots & \vdots & \vdots & \ddots \\
1 & 1 & 1 & \cdots
\end{array}\right) .
$$

Each set of initial condition $\phi_{\hat{i}}^{a}$ generates a solution with the incoming boundary condition, denoted by $\Phi_{\hat{i}}^{a}(r)$, which can be expanded near the boundary as following:

$$
\Phi_{\hat{i}}^{a}(r) \rightarrow \mathbb{S}_{\hat{i}}^{a}+\cdots+\frac{\mathbb{O}_{\hat{i}}^{a}}{r^{\delta_{a}}}+\cdots \quad \text { (near boundary) }
$$

where $\mathbb{S}_{\hat{i}}^{a}$ correspond to the sources, which are the leading terms of $\hat{i}$-th solution, and $\mathbb{O}_{\hat{i}}^{a}$ are relavent to the expectation values of the operators corresponding to the sources $\mathbb{S}_{\hat{i}}^{a}\left(\delta_{a} \geq 1\right)$. 
The general solution can be obtained as a linear combination of them. Let

$$
\Phi^{a}(r) \equiv \Phi_{\hat{i}}^{a}(r) c^{\hat{i}} \rightarrow \mathbb{S}_{\hat{i}}^{a} c^{\hat{i}}+\cdots+\frac{\mathbb{O}_{\hat{i}}^{a} c^{\hat{i}}}{r^{\delta_{a}}}+\cdots \quad \text { (near boundary) }
$$

with real constants $c^{i}$ 's. We want to choose $c^{\hat{i}}$ such that the leading term in the general solution $\left(i . e \Phi_{\hat{i}}^{a}(r) c^{\hat{i}}\right)$ is identified with the independent sources $J^{a}$. But if there are constraints, we need the additiononal set of solutions since $a>\hat{i}$. In this case, however, we can generate $n_{c}$ solutions by using residual gauge transformations.

In our case, $n=8$ and $n_{c}=2$, which corresponds to the constraints $g_{r i}=0$. There are two sets of additional constant solutions of the equations of motion (2.18)-(2.22)

$$
h_{t i}=h_{t i}^{0}, \quad a_{i}=-\frac{i H \epsilon_{i j} h_{t j}^{0}}{\omega}, \quad \phi_{i}=\frac{i \alpha h_{t i}^{0}}{\omega}, \quad \psi_{i}=\frac{i \beta h_{t i}^{0}}{\omega}
$$

where $h_{t i}^{0}$ is arbitrary constant and $i, j=x, y$. Therefore, the explicit expression for $\mathbb{S}_{\bar{i}}^{a}$ is

$$
\mathbb{S}_{\hat{i}}^{a}=\left(\mathbb{S}_{7}^{a} \mathbb{S}_{8}^{a}\right)=\left(\begin{array}{cc}
0 & -i \frac{H}{\omega} \\
i \frac{H}{\omega} & 0 \\
1 & 0 \\
0 & 1 \\
i \frac{\alpha}{\omega} & 0 \\
0 & i \frac{\alpha}{\omega} \\
i \frac{\beta}{\omega} & 0 \\
0 & i \frac{\beta}{\omega}
\end{array}\right)
$$

These can be understood as residual gauge transformations corresponding $g_{r i}=0$, which is generated by the vector field $\xi^{\mu}$ whose non-vanishing component is $\xi^{i}=\epsilon^{i} e^{-i \omega t}$ with constant $\epsilon^{i}$. So we add a vector along the residual gauge orbit. Since $\mathcal{L}_{\xi} g_{t i}=-i \omega r^{2} \xi^{i}$ and $\mathcal{L}_{\xi} \phi=\beta \xi^{i}, \mathcal{L}_{\xi} A_{i}=-H \epsilon_{i j} \xi^{j}$. Hence the most general solution becomes

$$
\Phi^{a}(r)=J^{a}+\cdots+\frac{R^{a}}{r^{\delta_{a}}}+\cdots,
$$

where we defined $J^{a}$ and $R^{a}$ as a source and response. For arbitrary sources $J^{a}$ we can always find $c^{I}$

$$
c^{I}=\left(\mathbb{S}^{-1}\right)_{a}^{I} J^{a}
$$

where $I=1, \ldots, n$. The corresponding response $R^{a}$ is expressed as

$$
R^{a}=\mathbb{O}_{I}^{a} c^{I}=\mathbb{O}_{I}^{a}\left(\mathbb{S}^{-1}\right)_{b}^{I} J^{b} .
$$

The general on-shell quadratic action in terms of the sources and the responses can be written as

$$
S_{\text {ren }}^{(2)}=\frac{\mathcal{V}_{2}}{2} \int_{0}^{\infty} \frac{d \omega}{2 \pi}\left[\bar{J}^{a} \mathbb{A}_{a b} J^{b}+\bar{J}^{a} \mathbb{B}_{a b} R^{b}\right]
$$


where $\mathbb{A}$ and $\mathbb{B}$ are regular matrices of order $n$. For example, we can rewrite the action (2.27) with:

$$
J^{a}=\left(\begin{array}{c}
a_{x}^{(0)} \\
a_{y}^{(0)} \\
h_{t x}^{(0)} \\
h_{t y}^{(0)} \\
\phi_{x}^{(0)} \\
\phi_{y}^{(0)} \\
\psi_{x}^{(0)} \\
\psi_{y}^{(0)}
\end{array}\right), \quad R^{a}=\left(\begin{array}{c}
a_{x}^{(1)} \\
a_{y}^{(1)} \\
h_{t x}^{(3)} \\
h_{t y}^{(3)} \\
\phi_{x}^{(3)} \\
\phi_{y}^{(3)} \\
\psi_{x}^{(3)} \\
\psi_{y}^{(3)}
\end{array}\right), \quad \mathbb{A}=\left(\begin{array}{cccc}
0 & -q & 0 & 0 \\
0 & -2 m_{0} & 0 & 0 \\
0 & 0 & 0 & 0 \\
0 & 0 & 0 & 0
\end{array}\right) \otimes \mathbf{1}_{2}, \quad \mathbb{B}=\left(\begin{array}{cccc}
1 & 0 & 0 & 0 \\
0 & -3 & 0 & 0 \\
0 & 0 & 3 & 0 \\
0 & 0 & 0 & 3
\end{array}\right) \otimes \mathbf{1}_{2},
$$

where $\mathbf{1}_{2}$ is the $2 \times 2$ unit matrix. Plugging the relation (2.37) into the action (2.38) we have

$$
S_{\text {ren }}^{(2)}=\frac{\mathcal{V}_{2}}{2} \int_{0}^{\infty} \frac{d \omega}{2 \pi} \bar{J}^{a}\left[\mathbb{A}_{a b}+\mathbb{B}_{a b} \mathbb{O}_{I}^{c}\left(\mathbb{S}^{-1}\right)_{b}^{I}\right] J^{b} .
$$

To sum up, we need four $n \times n$ matrices, $\mathbb{A}, \mathbb{B}, \mathbb{S}$ and $\mathbb{O}$ to compute the retarded Green's function. Each component of $\mathbb{A}$ and $\mathbb{B}$ can be read off from the quadratic on-shell action (2.27). The matrices $\mathbb{S}$ and $\mathbb{O}$ can be obtained by solving a set of the differential equations numerically. Notice that the choice of initial conditions is not relevant to the Green functions.

In our model, we can get a $8 \times 8$ matrix of the retarded Green functions. Here, however, we will focus only on the $4 \times 4$ sub-matrix corresponding to $a_{i}^{(0)}$ and $h_{t i}^{(0)}$ in $(2.25)$,

$$
\left(\begin{array}{cc}
G_{J J}^{i j} & G_{J T}^{i j} \\
G_{T J}^{i j} & G_{T T}^{i j}
\end{array}\right)
$$

where every $G_{A B}^{i j}$ is a $2 \times 2$ retarded Green's function with $i=x, y$ for given $A=J, T$. The lower indices $A, B$ denote the operators corresponding to the sources. i.e. the $a_{i}^{(0)}$ is a source of the electric current $J^{i}$ and the $h_{t i}^{(0)}$ is a source of the energy-momentum tensor $T^{t i}$. From the linear response theory, it is well known that response functions and the sources have the following relation:

$$
\left(\begin{array}{c}
\left\langle J^{i}\right\rangle \\
\left\langle T^{t i}\right\rangle
\end{array}\right)=\left(\begin{array}{cc}
G_{J J}^{i j} & G_{J T}^{i j} \\
G_{T J}^{i j} & G_{T T}^{i j}
\end{array}\right)\left(\begin{array}{c}
a_{j}^{(0)} \\
h_{t j}^{(0)}
\end{array}\right) .
$$

where $\left\langle J^{i}\right\rangle,\left\langle T^{t i}\right\rangle, a_{j}^{(0)}$ and $h_{t j}^{(0)}$ are $2 \times 1$ column matrices, with $i=x, y$. From this matrix relation, we want to obtain the electric $(\hat{\sigma})$, thermal $(\hat{\bar{\kappa}})$, and thermoelectric $(\hat{\alpha}, \hat{\bar{\alpha}})$ conductivities defined as

$$
\left(\begin{array}{c}
\left\langle J^{i}\right\rangle \\
\left\langle Q^{t i}\right\rangle
\end{array}\right)=\left(\begin{array}{cc}
\hat{\sigma}^{i j} & \hat{\alpha}^{i j} T \\
\hat{\bar{\alpha}}^{i j} T & \hat{\bar{\kappa}}^{i j} T
\end{array}\right)\left(\begin{array}{c}
E_{j} \\
-\left(\nabla_{j} T\right) / T
\end{array}\right) .
$$




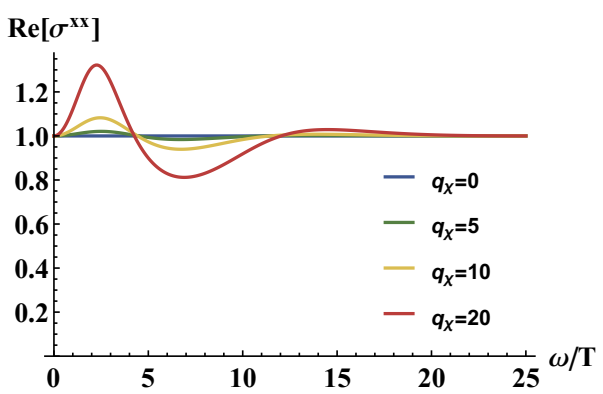

(a)

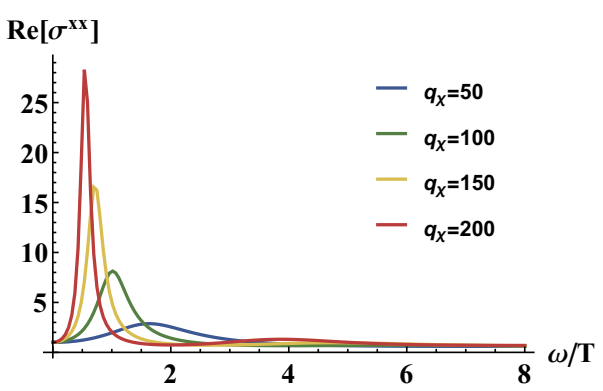

(b)

Figure 2. $q_{\chi}$ dependence of optical conductivity for $\mu=0$ and $H=0$. Stronger interaction gives sharper peak at smaller frequency and sum rule is checked.

where $E_{i}$ is an electric field, $\nabla_{i} T$ is a temperature gradient along the $i$ direction and $Q^{i}$ is a heat current, which is defined by $Q^{i}=T^{t i}-\mu J^{i}$. Notice that the electric and heat current here contain the contribution of magnetization which should be subtracted, so we use the conductivities with hat. By taking into account diffeomorphism invariance, (2.43) can be expressed as

$$
\begin{aligned}
\left(\begin{array}{cc}
\sigma^{i j} & \alpha^{i j} T \\
\bar{\alpha}^{i j} T & \bar{\kappa}^{i j} T
\end{array}\right)= & \left(\begin{array}{cc}
-\frac{i G_{J J}^{i j}}{\omega} & \frac{i\left(\mu G_{J J}^{i j}-G_{J T}^{i j}\right)}{\omega} \\
\frac{i\left(\mu G_{J J}^{i j}-G_{J T}^{i j}\right)}{\omega} & -\frac{i\left(G_{T T}^{i j}-G_{T T}^{i j}(\omega=0)-\mu\left(G_{J T}^{i j}+G_{T J}^{i j}-\mu G_{J J}^{i j}\right)\right)}{\omega}
\end{array}\right) \\
& -\frac{H}{T}\left(\begin{array}{cc}
0 & \Sigma_{1} \epsilon^{i j} \\
\Sigma_{1} \epsilon^{i j} & \Sigma_{2} \epsilon^{i j}
\end{array}\right)
\end{aligned}
$$

\section{Interaction induced quasi-particles in AC conductivity}

In this section, we investigate magnetic impurity effect on the optical conductivity. To see the effect of the magnetic impurity, we turn off the non-magnetic impurity density parameter, $\alpha=0$ in all calculations.

\subsection{Appearance of a new quasi particle at $\mu=H=0$}

As the first step to study $q_{\chi}$ coupling effect, we consider the simplest case where neither chemical potential nor external magnetic field exists. Here, the background geometry is simply the AdS Schwartzschild black hole with momentum relaxation. Then the value of $q_{\chi}$ term itself vanishes in the background solution, though its effect should appear in the fluctuation level as one can see in the Maxwell equation (2.20).

Figure 2 shows $q_{\chi}$ dependence of the optical conductivity. When $q_{\chi}=0$, the optical conductivity is constant, $\sigma(\omega)=1$, which is attributed to the electron-hole pair creation. When we turn on $q_{\chi}$, a quasi-particle peak appears as we can see from figure $2(\mathrm{a}, \mathrm{b})$. As we increase $q_{\chi}$, the peak becomes higher while the DC conductivity is kept to be one. See figure 2(a). We checked that the whole optical conductivity satisfies a sum rule, so that the total area below the optical conductivity curve is fixed. It means that the new quasiparticle degree of freedom is created at the price of reducing higher frequency modes. As 


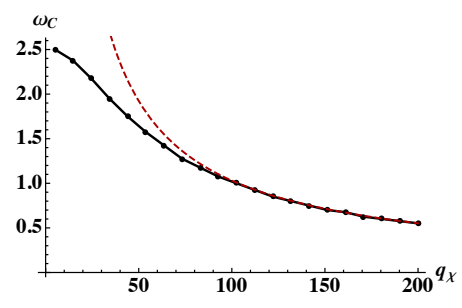

(a)

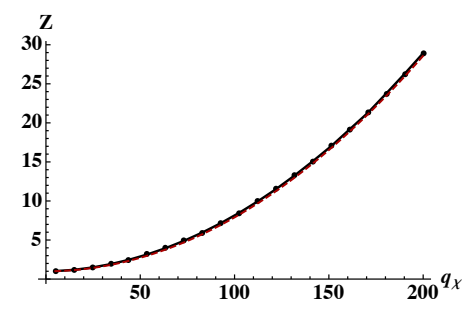

(b)

Figure 3. $q_{\chi}$ dependence of $\omega_{C}$ (peak frequency) and $Z$ (height of the peak). Dotted lines are $\omega_{C}=a q_{\chi}^{-1}(\mathrm{a})$ and $Z=b q_{\chi}^{2}+1(\mathrm{~b})$.

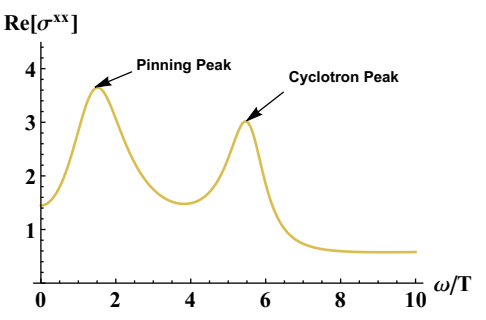

(a) Pinning and Cyclotron Peak

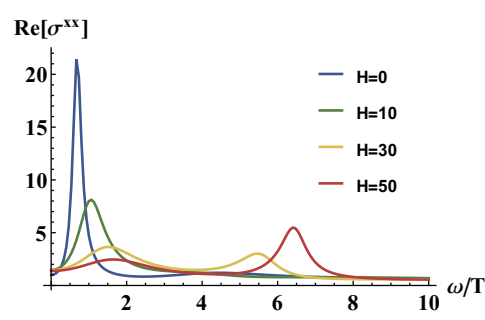

(b) $H$ evolution for $q_{\chi}=10$

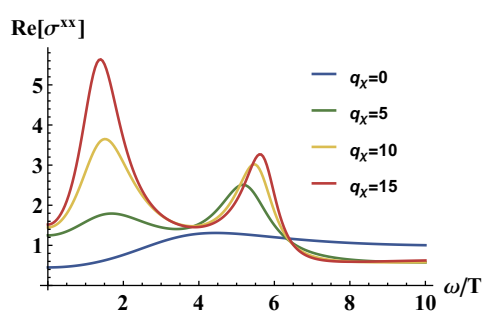

(c) $q_{\chi}$ evolution for $H=30$

Figure 4. Pinning peak v.s cyclotron peak: (a) Representative figure for pinning peak and cyclotron peak. $q_{\chi}=10$ and $H=30$. (b) $H$ evolution with fixed $q_{\chi}=10$. (c) $q_{\chi}$ evolution with fixed $H=30$.

we increase the value of $q_{\chi}$ further, the quasi-particle peak becomes sharper and the peak frequency $\left(\omega_{C}\right)$ is decreasing. See figure $2(b)$.

From the intuition of weakly interacting theory, this is surprising since one expects that interactions smooth out the particle character rather than create it. This feature of the optical conductivity is similar to the pinning effect [19, 20, 28-33] where charge density wave (CDW) is pinned by impurity so that only in a window of frequencies the charge density wave (CDW) can slide. Therefore, we will call this peak as 'pinning peak'. However, in our case the Drude peak does not disappear and just a new peak is created and therefore a metal-insulator transition does not follow.

The excitation frequency decreases as the interaction strength of impurity increases and the height of the peak $(Z)$ increases such that

$$
\omega_{C}=a q_{\chi}^{-1}, \quad Z=b q_{\chi}^{2}+1
$$

with some numerical constants $a$ and $b$. The $q_{\chi}$ dependence of $\omega_{C}$ and the height of the peak $Z$ are drawn in figure 3 .

\subsection{Pinning peak vs. Cyclotron peak: $\mu=0, H \neq 0$}

Now we study the effect of magnetic field on the optical conductivity in the absence of chemical potential. In our model, $\beta$ plays role of magnetic impurity density $q_{\chi}$ is its coupling with instanton density. One can ask whether the new peak in previous section, 


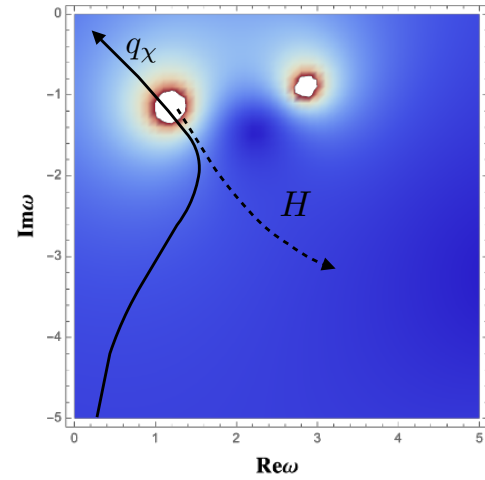

(a) Pinning pole

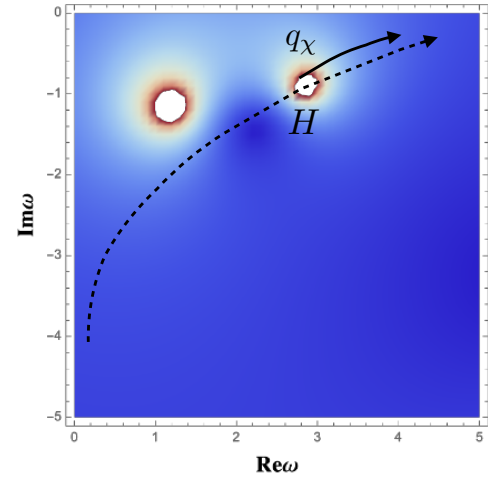

(b) Cyclotron pole

Figure 5. The evolution of the quasi-normal mode as $q_{\chi}$ (solid arrow) and $H$ (dashed arrow) increases. (a) For the pinning pole and (b) For the cyclotron pole. In both figures $\beta / T=3$, $q_{\chi}=10$ and $H / T^{2}=10$.

which we called as pinning peak, can be separated from the cyclotron peak which appears when one turns on the external magnetic field. The external magnetic field dependence of the cyclotron pole in the holographic context was already studied in [34, 35] before.

Figure 4(a) shows the pinning and cyclotron peak simultaneously. To see that these peaks are different, we study two cases i) change the value of $q_{\chi}$ at fixed $H$, ii) change the external magnetic field at the fixed coupling.

Figure 4(b) shows $H$-dependence of two peaks for fixed $q_{\chi}$. For $H=0$, only pinning peak exists as shown in the previous subsection. As we increase the external magnetic field, a new peak appears at higher frequency than that of the pinning peak. The frequency and the height of this peak increases as the external magnetic field is increased. This is the same behavior of the cyclotron peak in the dyonic black hole $[34,35]$. Therefore, we can say that the higher frequency peak is a cyclotron peak. Interestingly, as we increase the external magnetic field, the pinning peak is suppressed.

Figure 4(c) shows the $q_{\chi}$ dependence of the two peaks in the presence of the external magnetic field. For $q_{\chi}=0$ (blue line in the figure), there is a single cyclotron peak as it happens in the dyonic black hole. As we increase the value of $q_{\chi}$, the pinning peak is developed at lower frequency region. Simultaneously, the cyclotron peak gets also shaper. The latter phenomena can be understood as follows; the effect of $q_{\chi}$ gives anomalous magnetization, a finite magnetization in the absence of the external magnetic field $[4,5]$. Therefore, effective magnetic field increases as we increase $q_{\chi}$ at the fixed $H$. It makes the cyclotron peak sharper. Similar behavior happens in very large $q_{\chi}$ without external magnetic field.

One way to investigate pole structure is calculating $\sigma_{+} \equiv \sigma_{x y}+i \sigma_{x x}$ in complex $\omega$ plane [34]. Figure 5 shows density plot of $\left|\sigma_{+}\right|$at $\beta / T=3, q_{\chi}=10$ and $H=10$. For given parameters, there are two poles in complex $\omega$ plane. The pole nearer to the origin corresponds to the 'pinning pole'. The other pole is the cyclotron pole. Two arrowed lines indicate the movement of each pole as we increase $q_{\chi}$ (solid line) or $H$ (dashed line). The 


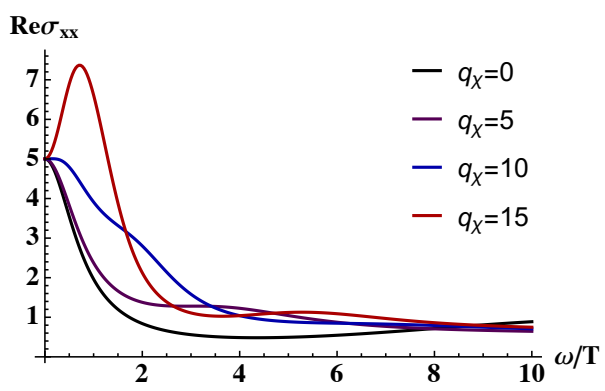

(a) $\mu / T=10, \beta / T=5$

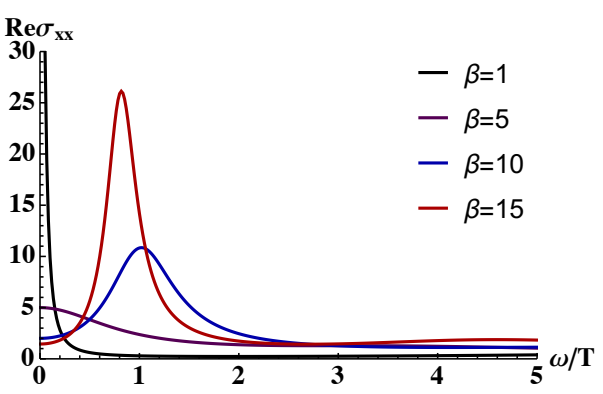

(b) $\mu / T=10, q_{\chi}=5$

Figure 6. (a) $q_{\chi}$ dependence of optical conductivity for $\mu / T=10, \beta / T=5$ and $H / T^{2}=0$. (b) $\beta$ dependence of optical conductivity for $\mu / T=10, q_{\chi}=5$ and $H=0$.

movement of the cyclotron peak is relatively simpler. It moves away from from the origin and approaches to the real line as we increases either $q_{\chi}$ or $H$. On the other hand, pinning pole moves in more complicated way under the increase of $q_{\chi}$ : it approaches to the real line but its real part increases at first but decreases after a critical point. Therefore, as $H$ increases, the cyclotron peak is getting sharper, but the pinning peak is getting fuzzier. As $q_{\chi}$ increases, both peaks are getting sharper.

\subsection{Drude vs. Pinning peak: $\mu \neq 0$ and $H=0$}

In this subsection, we investigate an effect of $\beta$ and $q_{\chi}$ with finite chemical potential. In the absence of $q_{\chi}$-term and the external magnetic field $H$, the background geometry becomes RN-AdS black hole with momentum relaxation. The optical conductivity in this background is studied in [26]. The optical conductivity changes from the coherent metalic state with the Drude peak to the incoherent state as the momentum relaxation parameter $\beta$ increases.

In the absence of the external magnetic field, the DC conductivity is independent of $q_{\chi}$ and remains the same as that of RN-AdS case [5] as one can see from

$$
\left.\sigma_{x x}\right|_{H=0}=1+\frac{\mu^{2}}{\beta^{2}} .
$$

Figure 6(a) shows $q_{\chi}$ evolution of the optical conductivity for given chemical potential and $\beta$. In this case, the DC conductivity is fixed by (3.2). As $q_{\chi}$ increased, the pinning peak is developed while the Drude peak does not changed. Similarly to the previous sections the pinning frequency decreases as $q_{\chi}$ increases and finally the Drude peak is observed. Figure 6(b) is $\beta$ dependence of the optical conductivity with fixed chemical potential and $q_{\chi}$. Here, the DC conductivity is suppressed by (3.2) and the pinning peak is also developed. The behavior of the pinning peak is similar to the evolution of $q_{\chi}$ because the $q_{\chi}$ interaction term in the action $(2.1)$ also contains scalar term $(\partial \chi)^{2}$ which is proportional to $\beta^{2}$. Therefore, increasing $\beta$ effect should be the same as increasing $q_{\chi}$.

One should notice, however, that the large $\beta$ behavior is different to large $q_{\chi}$. See figure 7 . For a large value of $q_{\chi}$, the peak frequency of the pinning peak is decreasing 


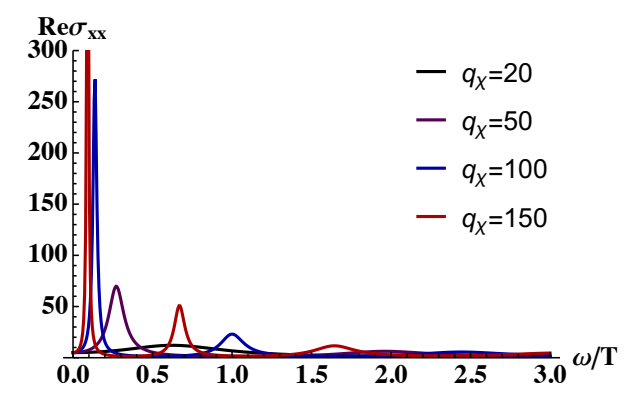

(a) $\mu / T=10, \beta / T=5$

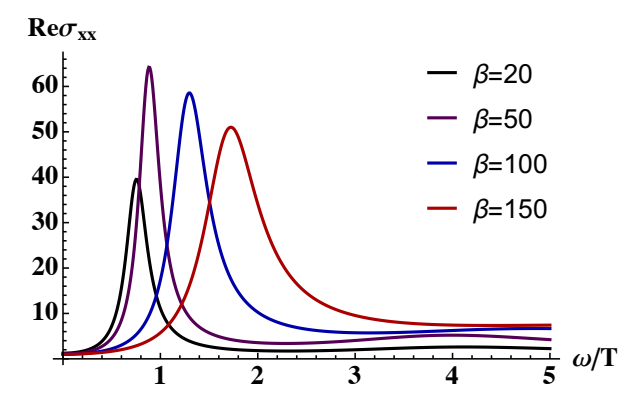

(b) $\mu / T=10, q_{\chi}=5$

Figure 7. (a) $q_{\chi}$ evolution of optical conductivity for large $q_{\chi} \cdot \mu / T=10, \beta / T=5$ and $H=0$. Notice that number of peaks increases as $q_{\chi}$ increases. (b) $\beta$ evolution of optical conductivity for large $\beta$. Notice that peak frequency increases as function of $\beta . \mu / T=10, q_{\chi}=5$ and $H=0$.

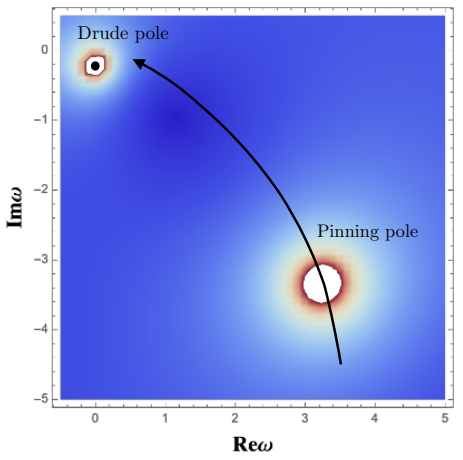

(a) $q_{\chi}$ evolution

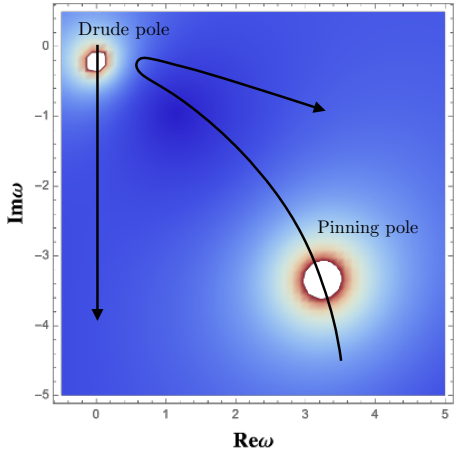

(b) $\beta$ evolution

Figure 8. (a) $q_{\chi}$ evolution of the Drude and the pinning pole. (b) $\beta$ evolution of the Drude and the pinning pole. The density plot is drawn with $\mu / T=5, \beta / T=2, q_{\chi}=5$ and $H=0$.

and the height is increasing. At the same time, another cyclotron peak appear at high frequency region as shown in 7(a). The appearance of new cyclotron peak comes from the finite magnetization effect with finite $q_{\chi}$. This magnetization gives effective magnetic field and hence a new cyclotron peak appear. On the other hand, when $\beta$ is increased, the pinning peak becomes sharper first (figure $6(\mathrm{~b})$ ). But if we increase $\beta$ more, the pinning peak is suppressed as figure $7(\mathrm{~b})$. This behavior can be understood as follows; $\beta$ plays a role of momentum relaxation parameter as well as a magnetic impurity via coupling term in the action. The pinning effect tends to make the peak sharp while the momentum relaxation effect tends to suppress the peak. Therefore, there is a competition between the pinning effect and the impurity effect. When $\beta$ is small, the pinning effect is stronger than the impurity effect and hence increasing $\beta$ is similar to increasing $q_{\chi}$. For a larger $\beta$, its effect is to suppress the pinning pole: the peak frequency goes high and the peak height is decreased. See figure 7(b).

Figure 8 is a density plot of $\left|\sigma_{+}\right|$at $\mu / T=5, \beta / T=1$ and $q_{\chi}=10$. When $\mu^{2} \gg \beta^{2}$, there is a sharp Drude peak in the optical conductivity. In complex $\omega$ plane, we call 'Drude pole' if the pole is near origin. The pinning pole is at finite complex $\omega$. Figure $8\left(\right.$ a) shows $q_{\chi}$ 
evolution of the Drude and the pinning pole. As $q_{\chi}$ increases the pinning pole approaches to the origin of the complex $\omega$ plane while the Drude pole does not move. In numerical calculation, the residue of the pinning pole is much larger than the Drude pole. Therefore, the Drude pole is almost overlapping with the pinning pole at large value of $q_{\chi}$ which is consistent with figure $6(\mathrm{a})$.

$\beta$ evolution of the Drude and the pinning pole is drawn in figure $8(\mathrm{~b})$. In the figure, the solid arrowed line denotes the direction of each pole as $\beta$ is increased. As $\beta$ is increased, the Drude pole move down along the imaginary $\omega$ axis which corresponds to the suppression of the real part of the conductivity. The $\beta$-evolution of the pinning pole is more non-trivial: at first, the pinning pole approaches to the origin just like the case of the $q_{\chi}$ evolution. But if we increase $\beta$ more, the pinning pole changes its direction to positive $\omega$ and the image on the real $\omega$ axis becomes figure $7(\mathrm{~b})$.

One remark is that there is a phase transition in $\beta$ evolution: for small $\beta$ the Drude peak is sharp therefore it is metal but for large values of $\beta$ the Drude pole is moved below so that it is insulator or bad metal at best. See figure 6(b). Therefore, describing the coupling dependence of the Drude peak is a good way to describe the Metal-insulator transition in the model.

\subsection{Origin of pinning pole}

Now it is time to discuss what the origin and nature of the new pinning peak are. Different interaction term can create a new pole by different mechanism. So we confine ourself to our specific mechanism. Since we look at the dynamics of current operators $J_{\mu}$ at the boundary, we consider their bulk dual field $A_{\mu}$. We first observe from the eq. (2.20) and eq. (2.18) that for the cyclotron peak $a_{x}$ is related to $a_{y}$ through the $h_{t y}$ to create a vortex, ${ }^{1}$ while for the pinning peak, $a_{x}, a_{y}$ are connected by the $q_{c h i}\left(\beta^{2}\right)$ term directly. Such difference is what makes the behaviors of the two poles different. In other words, the pinning pole is nothing but the cyclotron peak by the anomalous magnetic moment induced by the interaction term. In fact the term

$$
\epsilon_{i j}\left(\frac{2 i \theta \omega}{r^{3} U} a_{j}+\frac{i H \omega}{U^{2}} h_{t j}\right)
$$

in the eq. (2.20) shows the parallelism of $\theta a_{y}$ and $H h_{t y}$ regarding their coupling to $a_{x}$.

However, one can ask that if the pinning pole is nothing but the cyclotron vortex due to the anomalous magnetization, how two poles can be created. In fact, if we assume that only the magnetic induction $B$, which is the sum of magnetization $M$ and the current-induced magnetic field $H$, can couple to the charged particle, then there can not be two separate cyclotron poles. However, such splitting $B=M+H$ is possible only when the interaction is the minimal one $A_{\mu} J^{\mu}$ and the source $J^{\mu}$ can be split into charge's motional current and magnetization current: $J^{\mu}=J_{c}^{\mu}+J_{m}^{\mu}$ with $J_{m}^{\mu}=\partial_{\nu} M^{\nu \mu}$ for some antisymmetric $M^{\nu \mu}$, so

\footnotetext{
${ }^{1}$ The rise of the cyclotron frequency in the presence of magnetic field is well understood even in holographic context [36]. Our word 'vortex' is to take the analogy with the magnetic moment inside the uniform magnetic field.
} 
that the equation of motion can be rewritten as

$$
\partial_{\nu}\left(F^{\mu \nu}-M^{\mu \nu}\right)=J_{c}^{\mu} .
$$

Taking the space-space components of $F^{\mu \nu}$ and $M^{\mu \nu}$ as the $B$ and $M$, we get the splitting $B-M=H$. We want to answer why two poles should exist.

The basic point is that such canonical splitting is impossible when we add a nonminimal interaction like Chern-Simon term or $q_{\chi}$ type coupling we used. In that case, $M$ and $B$ can develop independent cyclotron peaks. In fact, for all recent studies on holographic pinning effect, the interactions considered were in the category of non-minimal interaction of gauge fields, where we expect the presence of pinning effects.

Our observation explains the origin of pinning peak not only for our case but also for all other case as well. For example, if we add Chern-Simon type interaction to induce the metal insulator transition [7], such appearance of new pole, which we call as pinning peak, is very often as it was observed in [9-12] with a few different types of interactions. Such shifted peak also arises when one calculate the holographic transport using the higher derivative terms, see [37].

Finally we comment that although the physics of pinning peak in our case is described as the cyclotron peak by the anomalous magnetization( $M$ for $H=0)$, for some other cases, it might be described as the physics of anomalous polarization by taking a timespace component of $F^{\mu \nu}, M^{\mu \nu}$ so that $E+P=D$.

\section{Discussion}

In this paper, we have shown that an interaction which usually destroys the (quasi) particle nature of the excitations, sometimes, plays the opposite role by creating a quasi particle spectral line(s) which was absent before adding the interaction term. There is a similar phenomenon in the insulator to metal transition (IMT) in holography. For IMT the creation of the peak at $\omega=0$ of optical conductivity or spectral function is involved, due to a bulk interaction term. This is common whatever the origin of that IMT is. All microscopic mechanism including interaction induced (Mott), impurity induced (Anderson) and structural change induced (Band) one are just a way of creating a pole near $\omega=0$.

One might worry that there could be some issue of instability by the large couplings we used. The study on dynamical instability is under progress. Notice that our solution is exact one that took care of the back reaction of the $q_{\chi}$ coupling and perturbations around it are linearly stable. The usual story of instability by CS term is because one starts with the background which does not take into account the presence of it. The coupling $q_{\chi}$ measures the deviation of our background from the usual Reisner-Nordstrom solution, not the deviation of the fluctuation from the background solution. Thus the coupling strength does not matter. Also our interaction term has too high engineering dimension and is irrelevant in IR limit. We speculate that such stability might be the key to change the role of the interaction from killing a pole to creating a pole.

We described the appearance of a sharp peak in holography, but it is an example of more general phenomena of spectral transfer in many-body systems. For any the- 
ory respecting unitarity, the changes of the spectrum distribution occur under the sum rule constraint so the spectral change is just rearrangement of the degrees of freedom. The conductor-insulator classification depends on whether or not the spectral peak going through $\omega=0$.

The bulk locality in holography is consequence of boundary non-locality [38]. The bulk local interaction may be useful to describe the global structure change or randomly distributed disorder as well as the Plankian dissipation in strange metal that apparently require the non-local interaction.

The bulk interaction also changes the character of the system and we expect that it would be interesting to utilize this to characterize a system and to match it to a realistic system in the future. In this regards, it would be interesting to examine all the possible lowest interaction terms of fermions and gauge fields and check whether each interaction term really generates new branch of quasi particle spectrums.

\section{Acknowledgments}

We would like to thank M. Baggioli for interesting comments on our first version. This work is supported by Mid-career Researcher Program through the National Research Foundation of Korea grant No. NRF-2016R1A2B3007687. YS is supported by Basic Science Research Program through NRF grant No. NRF-2016R1D1A1B03931443. The work of K.Y. Kim was supported by Basic Science Research Program through the National Research Foundation of Korea (NRF) funded by the Ministry of Science, ICT \& Future Planning (NRF-2017R1A2B4004810) and GIST Research Institute (GRI) grant funded by the GIST in 2019 .

Open Access. This article is distributed under the terms of the Creative Commons Attribution License (CC-BY 4.0), which permits any use, distribution and reproduction in any medium, provided the original author(s) and source are credited.

\section{References}

[1] N. Iqbal and H. Liu, Real-time response in AdS/CFT with application to spinors, Fortsch. Phys. 57 (2009) 367 [arXiv:0903.2596] [INSPIRE].

[2] M. Edalati, R.G. Leigh and P.W. Phillips, Dynamically generated Mott gap from holography, Phys. Rev. Lett. 106 (2011) 091602 [arXiv:1010.3238] [INSPIRE].

[3] Y. Seo, G. Song, Y.-H. Qi and S.-J. Sin, Mott transition with holographic spectral function, JHEP 08 (2018) 077 [arXiv:1803.01864] [INSPIRE].

[4] Y. Seo, K.-Y. Kim, K.K. Kim and S.-J. Sin, Character of matter in holography: spin-orbit interaction, Phys. Lett. B 759 (2016) 104 [arXiv: 1512.08916] [INSPIRE].

[5] Y. Seo, G. Song, C. Park and S.-J. Sin, Small Fermi surfaces and strong correlation effects in Dirac materials with holography, JHEP 10 (2017) 204 [arXiv:1708.02257] [INSPIRE].

[6] S. Nakamura, H. Ooguri and C.-S. Park, Gravity dual of spatially modulated phase, Phys. Rev. D 81 (2010) 044018 [arXiv:0911.0679] [InSPIRE]. 
[7] A. Donos and J.P. Gauntlett, Holographic charge density waves, Phys. Rev. D 87 (2013) 126008 [arXiv: 1303.4398] [INSPIRE].

[8] J. Erdmenger, X.-H. Ge and D.-W. Pang, Striped phases in the holographic insulator/superconductor transition, JHEP 11 (2013) 027 [arXiv: 1307.4609] [INSPIRE].

[9] N. Jokela, M. Jarvinen and M. Lippert, Gravity dual of spin and charge density waves, JHEP 12 (2014) 083 [arXiv: 1408.1397] [INSPIRE].

[10] Y. Ling, C. Niu, J. Wu, Z. Xian and H.-b. Zhang, Metal-insulator transition by holographic charge density waves, Phys. Rev. Lett. 113 (2014) 091602 [arXiv:1404.0777] [INSPIRE].

[11] A. Donos, B. Goutéraux and E. Kiritsis, Holographic metals and insulators with helical symmetry, JHEP 09 (2014) 038 [arXiv:1406.6351] [INSPIRE].

[12] R.G. Cai, L. Li, Y.Q. Wang and J. Zaanen, Intertwined order and holography: the case of parity breaking pair density waves, Phys. Rev. Lett. 119 (2017) 181601.

[13] A. Donos and J.P. Gauntlett, Holographic striped phases, JHEP 08 (2011) 140 [arXiv: 1106.2004] [INSPIRE].

[14] A. Donos and S.A. Hartnoll, Interaction-driven localization in holography, Nature Phys. 9 (2013) 649 [arXiv: 1212.2998] [INSPIRE].

[15] A. Donos, Striped phases from holography, JHEP 05 (2013) 059 [arXiv:1303.7211] [INSPIRE].

[16] B. Withers, Holographic checkerboards, JHEP 09 (2014) 102 [arXiv:1407.1085] [INSPIRE].

[17] R.-G. Cai, L. Li, Y.-Q. Wang and J. Zaanen, Intertwined order and holography: the case of parity breaking pair density waves, Phys. Rev. Lett. 119 (2017) 181601 [arXiv:1706.01470] [INSPIRE].

[18] T. Andrade, A. Krikun, K. Schalm and J. Zaanen, Doping the holographic Mott insulator, Nature Phys. 14 (2018) 1049 [arXiv:1710.05791] [INSPIRE].

[19] N. Jokela, M. Jarvinen and M. Lippert, Pinning of holographic sliding stripes, Phys. Rev. D 96 (2017) 106017 [arXiv: 1708.07837] [INSPIRE].

[20] T. Andrade, M. Baggioli, A. Krikun and N. Poovuttikul, Pinning of longitudinal phonons in holographic spontaneous helices, JHEP 02 (2018) 085 [arXiv:1708.08306] [INSPIRE].

[21] A. Donos, D. Martin, C. Pantelidou and V. Ziogas, Incoherent hydrodynamics and density waves, arXiv:1906.03132 [INSPIRE].

[22] L.V. Delacrétaz, B. Goutéraux, S.A. Hartnoll and A. Karlsson, Bad metals from fluctuating density waves, SciPost Phys. 3 (2017) 025.

[23] H. Fukuyama and P.A. Lee, Dynamics of the charge-density wave. I. Impurity pinning in a single chain, Phys. Rev. B 17 (1978) 535.

[24] G. Grüner et al., Frequency-dependent conductivity in nbse ${ }_{3}$, Phys. Rev. Lett. 45 (1980) 935.

[25] H. Fukuyama, Pinning and conductivity of two-dimensional charge-density waves in magnetic fields, Phys. Rev. B 18 (1978) 6245.

[26] K.-Y. Kim, K.K. Kim, Y. Seo and S.-J. Sin, Coherent/incoherent metal transition in a holographic model, JHEP 12 (2014) 170 [arXiv:1409.8346] [INSPIRE].

[27] T. Andrade and B. Withers, A simple holographic model of momentum relaxation, JHEP 05 (2014) 101 [arXiv:1311.5157] [INSPIRE]. 
[28] M. Baggioli and O. Pujolàs, Electron-phonon interactions, metal-insulator transitions and holographic massive gravity, Phys. Rev. Lett. 114 (2015) 251602 [arXiv:1411.1003] [INSPIRE].

[29] L. Alberte et al., Black hole elasticity and gapped transverse phonons in holography, JHEP 01 (2018) 129 [arXiv:1708.08477] [INSPIRE].

[30] M. Ammon, M. Baggioli and A. Jiménez-Alba, A unified description of translational symmetry breaking in holography, JHEP 09 (2019) 124 [arXiv: 1904.05785] [INSPIRE].

[31] W.J. Li and J.P. Wu, A simple holographic model for spontaneous breaking of translational symmetry, Eur. Phys. J. C 79 (2019) 243 [arXiv:1808.03142].

[32] A. Amoretti, D. Areán, B. Goutéraux and D. Musso, Universal relaxation in a holographic metallic density wave phase, arXiv:1812.08118 [INSPIRE].

[33] A. Donos and C. Pantelidou, Holographic transport and density waves, JHEP 05 (2019) 079.

[34] S.A. Hartnoll and C.P. Herzog, Ohm's law at strong coupling: $S$ duality and the cyclotron resonance, Phys. Rev. D 76 (2007) 106012 [arXiv:0706.3228] [INSPIRE].

[35] K.-Y. Kim, K.K. Kim, Y. Seo and S.-J. Sin, Thermoelectric conductivities at finite magnetic field and the Nernst effect, JHEP 07 (2015) 027 [arXiv: 1502.05386] [INSPIRE].

[36] S.A. Hartnoll, P.K. Kovtun, M. Muller and S. Sachdev, Theory of the Nernst effect near quantum phase transitions in condensed matter and in dyonic black holes, Phys. Rev. B 76 (2007) 144502 [arXiv:0706.3215] [INSPIRE].

[37] R.C. Myers, S. Sachdev and A. Singh, Holographic quantum critical transport without self-duality, Phys. Rev. D 83 (2011) 066017 [arXiv:1010.0443] [INSPIRE].

[38] A. Hamilton, D.N. Kabat, G. Lifschytz and D.A. Lowe, Holographic representation of local bulk operators, Phys. Rev. D 74 (2006) 066009 [hep-th/0606141] [InSPIRE]. 